\title{
Cardiotocography in early labour: a screening test for predicting fetal outcome
}

\author{
Vinothini Anandabaskar ${ }^{1}$, Hiremath P. B. ${ }^{1}$, Sanjeevkumar B. ${ }^{3 *}$, Nivedhana Arthi ${ }^{1}$, \\ Rathnapratheep R. ${ }^{2}$
}

\begin{abstract}
${ }^{1}$ Department of Obstetrics and Gynaecology, ${ }^{2}$ Department of Paediatrics, SVMCH and RC, Puducherry, India
${ }^{3}$ Department of Anaesthesiology, IGMC and RI, Puducherry, India
\end{abstract}

Received: 14 November 2020

Accepted: 15 December 2020

\section{*Correspondence:}

Dr. Sanjeevkumar B.,

E-mail: vinothinibaskar37@gmail.com

Copyright: () the author(s), publisher and licensee Medip Academy. This is an open-access article distributed under the terms of the Creative Commons Attribution Non-Commercial License, which permits unrestricted non-commercial use, distribution, and reproduction in any medium, provided the original work is properly cited.

\begin{abstract}
Background: Fetal surveillance during labour is important to ensure delivery of a healthy baby with minimum intervention. Various parameters can be used to assess the fetal wellbeing like fetal movements perceived by the mother and monitoring of fetal heart rate. The objective of this study is to evaluate the efficacy of cardiotocography in early labour in predicting fetal outcome.

Methods: A prospective hospital based cross sectional study was conducted on a total of 160 antenatal women in early labour with gestational age $\geq 37$ weeks of gestation. Cardiotocogram was recorded for 20 minutes with the antenatal women in left lateral position. They are followed up till delivery and the mode of delivery of the baby was recorded. After delivery of the baby, the presence of fetal distress was assessed by careful examination of the neonate and colour of liquor.

Results: Among the antenatal women, cardiotocography findings were normal in $74.4 \%$, suspicious in $15 \%$ and pathological in $10.6 \%$. The sensitivity of CTG in evaluating fetal outcome was found to be $84.6 \%$, and the specificity was found to be $85.8 \%$. The positive predictive value was $53.7 \%$ and the negative predictive value was $96.6 \%$.

Conclusions: Hence from the above study, it is can be concluded that the false positive rate of CTG is higher. Hence CTG cannot be used a sole indicator of fetal hypoxia. But in a resource limited setting, CTG is definitely a good screening test to predict the fetal outcome.
\end{abstract}

Keywords: APGAR score, Cardiotocography, Decelerations, Fetal hypoxia, Lower segment caesarean section, Meconium stained liquor

\section{INTRODUCTION}

Fetal surveillance during labour is important to ensure delivery of a healthy baby with minimum intervention. Various parameters can be used to assess the condition of the fetus like fetal movements perceived by the mother and monitoring of fetal heart rate. Ingemarsson et al described monitoring fetal heart rate during labour to identify apparently normal women whose fetus were compromised in labour. ${ }^{1}$ Admission test was used in early labour to detect compromised fetus on admission to the labour ward and to select women in need of continuous electronic fetal monitoring during labour. The uterine contractions of labour causes stress on placental circulation so any abnormal tracing in latent labour helps to identify fetal compromise at an early stage to allow timely intervention. ${ }^{2}$ Routine electronic monitoring of fetal heart rate has become an established practice in developed countries, although in developing countries its use has been restricted to high risk patients due to financial constraints. 
The main aim of cardiotocography during labour is to identify fetal hypoxia at an early stage, thus preventing fetal acidemia and hypoxic ischemic encephalopathy of the neonate. ${ }^{3}$

Fetal hypoxia and acidemia in severe cases leads to irreversible brain damage to the fetus resulting in hypoxia ischaemic encephalopathy and cerebral palsy. These serious complications can be avoided, if fetal distress is identified at an early stage and timely intervention is given. Hence cardiotocography plays a major role in labour monitoring and identification of fetal distress.

\section{METHODS}

A prospective hospital based cross sectional study was conducted on a total of 160 antenatal women attending the Obstetrics outpatient department at Sri Venkateshwaraa Medical College, Hospital and Research Centre, Puducherry for a period of 16 months.

Antenatal women giving written informed consent will be selected for the study. On admission, details like age, obstetric, medical and menstrual history will be documented. General examination, per abdomen and per vaginal examinations will be done and the stage of labour will be determined. All routine investigations like haemoglobin, blood grouping and typing, random blood sugar and urine routine examination and other relevant investigations in case of high risk pregnancies will be documented. Ultrasound findings will be documented. Lethal congenital malformations will be ruled out using the ltrasound findings available.

\section{Inclusion criteria}

Pregnant women more than or equal to 37 weeks period of gestation with cephalic presentation in early labour.

\section{Exclusion criteria}

Multiple gestation. Acute fetal hypoxic states like abruption, cord prolapse, uterine scar rupture etc. requiring immediate termination of pregnancy. Any lethal congenital malformation detected on ultrasonography. Antenatal women identified for elective LSCS (Lower segment caesarean section).

Cardiotocogram will be recorded with the cardiotocograph machine model UT3000 which runs at the speed of $3 \mathrm{~cm}$ per minute, for 20 mins with the antenatal women in left lateral position. The five components calculated from the tracings include baseline fetal heart rate, beat to beat variability, fetal heart rate accelerations, decelerations and sinusoidal pattern. The decelerations, if present will be categorized into early, late and variable deceleration based on its association with uterine contractions. In case of absent beat to beat variability for 20mins CTG is continued for 40mins or if needed upto 90 minutes. The fetal heart rate traces will thus be categorized into normal, suspicious and pathological CTG according to NICE guidelines. ${ }^{3}$

The patients are followed up till delivery and the mode of delivery of the baby will be recorded. After delivery of the baby, the presence of fetal distress will be assessed by careful examination of the neonate and colour of liquor. Fetal distress will be indicated by the presence of any one of the following parameters:

Moderate to thick Meconium Stained Liquor, Apgar score of neonate at 1 min less than 74 , NICU admission for Birth asphyxia, Intrapartum or neonatal mortality.

The parameters of fetal distress and the mode of delivery will be compared among the various CTG categories (normal, suspicious and pathological).

\section{Statistical analysis}

The data was entered in excel sheet and analyzed using Statistical Package for Social Sciences (SPSS-version 17). Descriptive statistics with mean, standard deviation and proportions (\%) were calculated for continuous variables. Chi square test, Odds ratio, 95\% confidence interval $(\mathrm{CI})$ and the diagnostic test parameters were calculated to test the hypothesis. A p value $<0.05$ was considered as statistically significant.

\section{RESULTS}

In this study among 160 antenatal women, the mean age of the study participants was $25.6 \pm 4.3$ years. Among them, $57.5 \%$ of the antenatal women were multigravida and $42.5 \%$ of the antenatal women were primigravida.

In this current study, risk factor during pregnancy like anaemia was found to be present in $22.5 \%$ of the study participants, history of previous LSCS was seen among $19.3 \%$ of the participants, gestational hypertension and oligohydramnios was found to be present in $11.3 \%$ and $10.6 \%$ of the antenatal women respectively. Risk factor like hypothyroidism was seen in $9.4 \%$ of the study participants and gestational diabetes mellitus was found among $8.8 \%$ of the study women. About $3.1 \%$ of the women had bad obstetric history and $3.1 \%$ of the women were $\mathrm{Rh}$ negative pregnancy and another $3.1 \%$ were teenage pregnancy.

Among the entire participants, $2.5 \%$ of them were found to have cephalopelvic disproportion. Other risk factors like Intra Uterine Growth Restriction, cervical incompetence and imminent eclampsia was noted among $0.6 \%$ of the antenatal women in our study. Psychosis, seizure disorder, elderly primigravida, dextrocardia and obesity was also found to be $0.6 \%$ in this study.

In this current study risk factors were present among $72.5 \%$ of the participants of whom $55 \%$ had normal CTG, $7.5 \%$ had pathological CTG and $10 \%$ of them had 
suspicious CTG recordings. Among 27.5\% participants with no risk factors $19.4 \%$ had normal CTG recording, $3.1 \%$ had pathological CTG recording and $5 \%$ of the participants had suspicious CTG recording.

In the present study, $91.9 \%$ of the fetuses had normal FHR, while $6.9 \%$ of the foetuses were found to have tachycardia and $1.3 \%$ of the foetuses were found to have bradycardia at baseline examination.

Based on the CTG findings, beat to beat variability was present in $83.1 \%$ of the participants in a 20 minute tracing. In this current study, beat to beat variability was well observed in $96.9 \%$ of the study participants within 90 minutes but was not present among $3.1 \%$ of the study participants.

Early decelerations were noted among $3.1 \%$ of the study participants. Late decelerations were recorded among $5.6 \%$ of the study. Variable decelerations were seen in $4.4 \%$ of the antenatal women. Sinusoid fetal heart rate was not recorded in any of the antenatal women in our study.

Among the study participants, cardiotocography findings were normal in $74.4 \%$ of them. Suspicious cardiotocography findings were observed in $15 \%$ of the participants whereas $10.6 \%$ of the participants had pathological cardiotocography finding.

\section{Table 1: Proportion of participants based on} cardiotocographic findings.

\begin{tabular}{|lll|}
\hline $\begin{array}{l}\text { Cardiotocography } \\
\text { findings }\end{array}$ & Frequency & Percentage \\
\hline Normal & 119 & 74.4 \\
\hline Pathologic & 17 & 10.6 \\
\hline Suspicious & 24 & 15.0 \\
\hline Total & 160 & 100.0 \\
\hline
\end{tabular}

Among 160 women, the mode of present delivery was LSCS for $49.4 \%$ participants, NVD for $43.1 \%$ of the participants, forceps delivery for $3.1 \%$ of the participants and vacuum for $4.4 \%$ of the study participants.

Table 2: Mode of delivery among the study participants.

\begin{tabular}{|lll|}
\hline Mode of Delivery & Frequency & Percentage \\
\hline LSCS & 79 & 49.4 \\
\hline NVD & 69 & 43.1 \\
\hline Forceps & 5 & 3.1 \\
\hline Vacuum & 7 & 4.4 \\
\hline Total & 160 & 100.0 \\
\hline
\end{tabular}

In this current study, liquor was clear in $72.5 \%$ of the participants. Among remaining 27.5 of the participants, $18.2 \%$ of them had thin Meconium stained liquor and $9.3 \%$ of the women had thick Meconium stained liquor.
Among $90.6 \%$ of the participants with clear liquor $73.89 \%$ of them had a normal CTG while $16.9 \%$ of them had abnormal CTG. Among $9.4 \%$ of the study participants with thick stained Meconium $0.6 \%$ of them had normal CTG and $8.8 \%$ of them were found to have abnormal CTG findings.

The 1 minute APGAR score of 2, 3, 4 and 5 was observed among $1.3 \%, 1.3 \%, 3.1 \%$ and $4.4 \%$ of the newborns respectively. APGAR score of 6 was observed in $4.4 \%$ of them whereas $14.4 \%$ of them had an APGAR score of 7 . About $59.4 \%$ of the newborns had APGAR score of 8 and a score of 9 was seen in $11.9 \%$ of them. Among $85.6 \%$ of the newborns with normal APGAR score at 1 minute, $72.5 \%$ of the newborn had normal CTG recording $13.1 \%$ of them were found to have abnormal CTG.

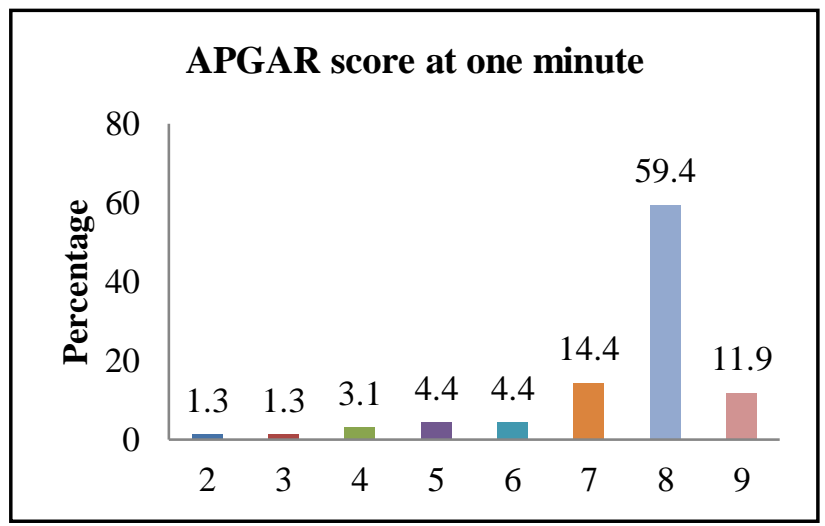

Figure 1: Proportion of participants based on APGAR score at one minute.

Among all the newborns, $13.1 \%$ of them required Neonatal Intensive Care (NICU) admission for birth asphyxia. In this present study there was no neonatal mortality recorded. Based on the CTG categories, $74.4 \%$ of the participants were found to have normal CTG and $25.6 \%$ had abnormal CTG findings.

Table 3: CTG Vs fetal distress among the study participants.

\begin{tabular}{|llll|}
\hline \multirow{2}{*}{ CTG } & \multicolumn{2}{l}{ Fetal Distress } & Total \\
\hline Abnormal & Present & Absent & \\
\hline Normal & 22 & 19 & 41 \\
\hline Total & 4 & 115 & 119 \\
\hline
\end{tabular}

Table 4: Efficacy of the screening test- CTG.

\begin{tabular}{|lll|}
\hline Variables & Percentage & 95\% CI \\
\hline Sensitivity & 84.6 & $65.1-95.6$ \\
\hline Specificity & 85.8 & $78.8-91.2$ \\
\hline Positive predictive value & 53.7 & $42.5-64.4$ \\
\hline Negative predictive value & 96.6 & $92.1-98.6$ \\
\hline
\end{tabular}


The sensitivity of CTG in identifying fetal distress was found to be $84.6 \%$, and the specificity of CTG in this study was found to be $85.8 \%$. The positive predictive value of CTG according to this study is $53.7 \%$ and the negative predictive value is $96.6 \%$.

\section{DISCUSSION}

Cardiotocography is a technique of fetal surveillance in labour that identifies fetal hypoxia at an early stage allowing timely intervention. Thus reducing the incidence of irreversible hypoxic brain damage to the fetus. A high inter-observer variability in the interpretation of CTG was overcome by standardising the interpretation of the CTG tracing.

The CTG tracings are categorized as normal, suspicious and pathological according to RCOG guidelines. ${ }^{3}$ In our study $74.4 \%$ had normal CTG, $10.6 \%$ had pathological CTG whereas $15 \%$ had suspicious CTG. Fetal distress was identified by the presence of moderate to thick meconium staining of liquor, APGAR score, NICU admission, intrapartum or neonatal mortality.

Among 41 women with abnormal CTG, fetal distress was present in 22 of them whereas among 119 women with normal CTG, fetal distress was present in 4 of them. The sensitivity of CTG in evaluating fetal outcome was found to be $84.6 \%$, and the specificity of CTG was found to be $85.8 \%$. The positive predictive value of CTG according to the study is $53.7 \%$ and the negative predictive value of CTG is $96.6 \%$. This is in accordance to a study conducted by Sandhu et al, in which foetal distress during labour was reported among $15 \%$ of cases with a normal CTG and in $73 \%$ of cases with an abnormal CTG. ${ }^{5}$ CTG test had a positive predictive value of $53.3 \%$ for predicting an APGAR score $<5$ at birth.

In this present study, among women with normal CTG, fetal distress was noted in $2.5 \%$ of the fetus and among women with suspicious CTG, $7.5 \%$ of them had fetal distress. In participants with pathological CTG, $6.2 \%$ of them had fetal distress. The $\mathrm{p}$ value was found to be highly statistically significant. In another study, authors found a high specificity $(84.5 \%)$ and high negative predictive value $(98.9 \%)$ in predicting fetal distress. The positive predictive value of admission test in their study was similarly as low as $23.8 \%$; meanwhile, their sensitivity was higher, at up to $83.3 \%$. In their study, they reached a sensitivity of $42.3 \%$, specificity of $95.6 \%$ and positive predictive value of $73.3 \%$ for the prediction of 5min Apgar score $<7$. $^{6}$

Ducey et al reported that screening FHR tracing in the admitting room had a sensitivity of $57 \%$, specificity of $98 \%$, positive predictive value of $75 \%$ and overall efficiency of $95 \%$, in their study. ${ }^{7}$ Khandelwal et al found that patients with nonreactive traces had higher incidence of meconium staining, clinically detected fetal distress and operative delivery or caesarean section. ${ }^{8}$ Besides, incidence of poor neonatal outcomes was greater in both low- and high risk groups when compared with reactive traces. They found similar results to our study regarding the high specificity $(94.7 \%)$ and negative predictive value $(81.8 \%)$ in predicting clinically detected fetal distress.

In this current study, risk factors were present among $72.5 \%$ of the participants, of whom $55 \%$ had normal CTG, $7.5 \%$ had pathological CTG and $10 \%$ of them had suspicious CTG recordings. Sharbaf et al reported that $60 \%$ of cases were categorized as low-risk and $40 \%$ as high-risk pregnancies based on history and examination. In low-risk patients, $83.3 \%$ had normal CTG and $16.7 \%$ had indeterminate CTG. ${ }^{9}$

In this study, among $85.6 \%$ of the newborns with normal APGAR score at 1 minute $72.5 \%$ of the newborn had normal CTG recording but $13.1 \%$ of them were found to have abnormal CTG. Dellinger et al reported caesarean delivery for fetal distress was significantly higher in abnormal CTG group. ${ }^{10}$ Also they reported APGAR score was $<7$ at 1 min among the babies of abnormal CTG group than normal CTG. Druzin et al in his study found that bradycardia was associated with increased morbidity and mortality; the causes were cord compression, IUGR and fetal malformation. ${ }^{11}$

In our study, among $9.4 \%$ of the study participants with thick stained meconium, $0.6 \%$ of them had normal CTG and $8.8 \%$ of them were found to have abnormal CTG findings. According to Rahman et al, the incidence of moderate to thick meconium stained liquor was significantly high in ominous $(66.7 \%)$ and equivocal group $(18.2 \%)$, as compared to reactive group $(2.4 \%){ }^{2}$ Thick meconium staining of liquor is a highly suggestive indicator of fetal distress.

Chua et al reported that operative delivery for fetal distress, 5-min Apgar score <7, assisted ventilation and admission to NICU were significantly associated with suspicious or ominous CTG at admission. ${ }^{12}$ Elimian et al stated that patients in the non-reactive group were six times as likely to have fetal distress, and that fetuses with distress were more likely to have a non-reactive admission test. ${ }^{13}$ They found the fetal admission test to be useful in low-risk populations.

Dwarakanth et al reported incidence of reactive trace was $69 \%$, suspicious $24 \%$ and ominous $7 \% .{ }^{14}$ Among them $80 \%$ cases had vaginal delivery, $14 \%$ had LSCS and $6 \%$ had instrumental delivery. Sowmyashree et al reported $82 \%, 10 \%$ and $8 \%$ had normal, suspicious and pathological admission test result respectively. ${ }^{15}$

The incidence of foetal distress (100\% Vs 7.92\%), caesarean section rate (100\% Vs 7.3\%), low Apgar score at $5^{\prime}$ (50\% Vs $4.87 \%$ ), NICU admission (50\% Vs $4.87 \%$ ) was higher in pathological CTG group than in normal CTG group. 


\section{CONCLUSION}

The sensitivity of CTG in evaluating fetal outcome was found to be $84.6 \%$, and the specificity was found to be $85.8 \%$. The positive predictive value was $53.7 \%$ and the negative predictive value was $96.6 \%$.

In participants with pathological CTG, $6.2 \%$ of them had fetal distress. The probability of participants to get abnormal fetal outcome while CTG is abnormal is $53.7 \%$ and the probability of the participants not ending up with abnormal fetal outcome when CTG is normal was $96.6 \%$. Hence from the above study, it is can be concluded that the false positive rate of CTG is higher. Hence CTG cannot be used as a sole indicator of fetal hypoxia. But in a resource limited setting, CTG is definitely a good screening test to predict the fetal outcome.

\section{Funding: No funding sources}

Conflict of interest: None declared

Ethical approval: The study was approved by the Institutional Ethics Committee

\section{REFERENCES}

1. Ingemarsson I, Arulkumaran S, Ingemarsson E, Tambyraja RL, Ratnam SS. Admission test: A screening test for fetal distress in labour. Obstet Gynecol. 1986;68:800-6.

2. Rahman H, Renjhen P, Dutta S. Reliability of admission cardiotocography for intrapartum monitoring in low resource setting. Niger Med J. 2012;53:145-9.

3. Evidence-based clinical guideline No.8. The use and interpretation of CTG in intrapartum fetal surveillance.London. RCOG Press, 2001.

4. WHO. International Statistical Classification of Diseases and Related Health Problems (International Classification of Diseases) (ICD) 10th Revision Version:2010. Occupational Health. 2010.

5. Sandhu GS, Raju R, Bhattacharyya TK. Admission cardiotocography screening of high risk obstetric patients. Medical Journal Armed Forces India. 2008;64(1):43-5.
6. Sarno A, Ahn M, Brar H, Phelan J, Platt L. Intrapartum Doppler velocimetry, amniotic fluid volume, and fetal heart rate as predictors of subsequent fetal distress. I. An initial report. Am J Obstet Gynecol. 1989;161(6 Pt 1):1508.

7. Ducey J, Guzman E, Schulman H. Value of screening fetal heart rate tracing in the latent phase of labor. J Reprod Med. 1990;35:899-900.

8. Khandelwal S, Dhanaraj $M$, Khandelwal A. Admission test as precursor of perinatal outcome: A prospective study. Arch Gynecol Obstet. 2010;282:377-82.

9. Sharbaf FR, Amjadi N, Alavi A, Akbari S, Forghani F. Normal and indeterminate pattern of fetal cardiotocography in admission test and pregnancy outcome. J Obstetrics Gynaecol Res. 2014;40(3):694-9.

10. Dellinger EH, Boehm FH, Crane MM. Electronic fetal rate monitoring: Early neonatal outcomes associated with normal rate, fetal stress, and fetal distress. Am J Obs Gynecol. 2000;182:214-20.

11. Druzin ML, Fox A, Koght E. The relationship of the non stress test to gestational age. Am J Obs Gynecol. 1985; $153: 386$.

12. Chua S, Arulkumaran S, Kurup A, Anandakumar C, Selemat N, Ratnam SS. Search for the most predictive tests of fetal well-being in early labor. J Perinat Med. 1996;24:199-206.

13. Elimian A, Lawlor P, Figueroa R, Wiencek V, Garry D, Quirk J. Intrapartum assessment of fetal wellbeing: Any role for a fetal admission test? J Matern Fetal Neonatal Med. 2003;13:408-13.

14. Dwarakanath L, Lakshmikantha G, Chaitra SK. Efficacy of admission cardiotocography (admission test) to predict obstetric outcome. J Evolution Medic Dent Sci. 2013;2(5):418-24.

15. Thimmappa S, Datti SN. Use of Cardiotocogram (CTG) as an Admission Test as a Predictor of Foetal Outcome in Labour in Low Risk Group. J Evolution Medic Dent Sci. 2019;8(38):2911-5.

Cite this article as: Anandabaskar V, Hiremath PB, Sanjeevkumar B, Arthi N, Rathnapratheep R.

Cardiotocography in early labour - a screening test for predicting fetal outcome. Int J Reprod Contracept Obstet Gynecol 2021;10:321-5. 